\title{
High-grade MRI bone oedema is common within the surgical field in rheumatoid arthritis patients undergoing joint replacement and is associated with osteitis in subchondral bone
}

\author{
F M McQueen, A Gao, M Østergaard, A King, G Shalley, E Robinson, A Doyle, B Clark, N Dalbeth
}

Ann Rheum Dis 2007;66:1581-1587. doi: 10.1136/ard.2007.070326

See end of article for authors' affiliations

Correspondence to: F M McQueen, Associate Professor in Rheumatology, Department of Molecular Medicine and Pathology, Faculty of Medicine and Health Sciences, University of Auckland, Park Rd, Private Bag 92019

Auckland, New Zealand; f.mcqueen@auckland.ac.nz

Accepted 19 April 2007

\begin{abstract}
Objectives: MRI bone oedema has been observed in early and advanced RA and may represent a cellular infiltrate (osteitis) in subchondral bone. We studied MRI scans from RA patients undergoing surgery, seeking to identify regions of bone oedema and examine its histopathological equivalent in resected bone.

Methods: Preoperative contrast-enhanced MRI scans were obtained in 11 RA patients scheduled for orthopaedic surgery to the hands/wrists or feet. In 9, MRI scans were scored by 2 readers for bone oedema (RAMRIS system). Its distribution with respect to surgical site was investigated. In 4 patients, 7 bone samples were examined for a cellular infiltrate, and this was compared with MRI bone oedema, scored for spatial extent and intensity.

Results: Inter-reader intraclass correlation coefficients for bone oedema were 0.51 (all sites) and 0.98 (bone samples for histology). Bone oedema was observed at $60 \%$ of surgical sites vs $38 \%$ of non-surgical sites. High-grade bone oedema (score $\geqslant 50 \%$ maximum) was strongly associated with the surgical field (OR 9.3 (3.5 to 24.2), $p<0.0001)$. Bone oedema scores correlated with pain $(r=0.67, p=0.048)$ and CRP $(r=0.86$, $p=0.01$ ). In 4 of the 7 bone samples, there was concordance between bone oedema and subchondral osteitis. In 3, there was no MRI bone oedema, and osteitis was "slight".

Conclusion: High-grade MRI bone oedema was common within the field of intended surgery and associated with pain. There was concordance between the presence and severity of MRI bone oedema and osteitis on histology, with an MRI threshold effect due to differences in image resolution.
\end{abstract}

$\mathrm{T}$ e rate of erosive progression in rheumatoid arthritis (RA) is highly variable, and this disease ranges from a mild, indolent form to a rapidly disabling, erosive form that may result in patients requiring joint replacement or fusion within a few years of onset. ${ }^{1}$ Bone oedema is detectable on magnetic resonance imaging (MRI) scans as early as 9 weeks from the onset of symptoms ${ }^{2}$ and is a predictor of radiographic joint damage $^{3}$ and physical function ${ }^{4}$ in RA patients. A recent study revealed bone oedema to reflect severe disease status in patients with early-stage RA. ${ }^{5}$ These findings imply that bone oedema represents a fundamental part of the articular pathophysiology of RA and suggest it is most likely to be localised where active bony destruction is occurring.

Reports are now emerging, describing a cellular infiltrate or "osteitis" involving subchondral bone as the histopathological correlate of MRI bone oedema in RA. ${ }^{6}$ Some of these cells are contained within invading synovial tissue, and some are within lymphocytic aggregates. These findings were first reported in the early 1980s by Barrie et al, who described osteitis in 35\% of patients undergoing metatarsal head resection. ${ }^{7}$ More recently, Bugatti et $\mathrm{al}^{8}$ published a similar immunohistochemical study of RA joints and found lymphoid aggregates, often associated with osteoclasts, on the subchondral side of the joint in established RA. Furthermore, Proulx et al have now reported a close correlation between MRI bone oedema and a cellular infiltrate within subchondral bone in an animal model of RA. ${ }^{9}$ It has been proposed that this cellular infiltrate, visualised as bone oedema on MRI scans, could represent an important part of the immunopathology of RA, separate to but often occurring with synovial inflammation. ${ }^{10}$
The current study was designed to investigate whether MRI bone oedema was present at the site of surgery in RA patients undergoing joint replacement or fusion and whether it corresponded with the presence of osteitis in specimens of subchondral bone that might be resected.

\section{PATIENTS AND METHODS}

Patient population and clinical assessments

From February 2005 to December 2006, 11 patients fulfilling criteria for RA, ${ }^{11}$ scheduled for orthopaedic surgery to the hands/wrists or feet, were recruited from the Auckland region according to local ethics committee requirements. Clinical assessments and MRI scans were performed, 1-2 days prior to surgery. Surgeons were unaware of MRI findings when deciding the area for surgery. Patients were assessed using 66 tender and 68 swollen joint counts, pain score, erythrocyte sedimentation rate (ESR), C-reactive protein (CRP) and 3variable disease activity score (DAS), ${ }^{12}$ on the MRI scan day. Pre- and postoperative radiographs were obtained.

\section{Definition of the surgical field}

Any resected bone was included within the surgical field. Where joint fusion was performed, bone sites on either side were included. Where joint replacements were performed, bone proximal and distal to the joint was included. The same

Abbreviations: CRP, C-reactive protein; ICC, intraclass correlation coefficients; MRI, magnetic resonance imaging; RA, rheumatoid arthritis; RAMRIS, rheumatoid arthritis MRI scoring 
procedure was followed for defining whether joints lay within the surgical field.

\section{MRI}

Preoperative MRI scans were obtained using a 1.5-T scanner (Siemens) with dedicated extremity coil. Sequences were: coronal and axial Tl-weighted (Tlw), axial T2-weighted (T2w) fat-suppressed (FS) fast spin echo, coronal Tlw FS post gadolinium diethylenetriamine pentaacetic acid (Gd-DTPA), axial FS Tlw post-Gd-DTPA. The field of view was localised to the surgical field (14 to $24 \times 3 \mathrm{~mm}$ slices, specifications on request) where 2-plane information was available. Outside the surgical zone, 1-plane (coronal $\mathrm{TlW}$ and $\mathrm{T} 2 \mathrm{~W}$ sequences) information was available.

\section{Scoring MRI scans \\ Part 1}

MRI scans from 9 patients entered into Part 1 of the study were scored separately using the Outcome Measures in Rheumatology (OMERACT) RAMRIS system ${ }^{13}$ for synovitis, bone oedema and erosion by 2 rheumatologists, experienced in assessing MRI images of RA joints, from different institutions. One observer was blinded to the history of intended surgery. The number of bony sites assessed for bone oedema and erosion at the wrist, hand and foot were 15, 33 and 42 . Similarly, 3, 14 and 23 sites were assessed for synovitis, respectively. Data comprised mean scores for the 2 readers. Two analyses examined the influence of missing data. First, where one reader did not score a site because of distortion of anatomy or artefact, data from the one active reader were used. Second, this site was disqualified and not incorporated into the analysis. Results did not differ (data available on request), and the first analysis is presented here.

\section{Part 2}

For the comparison between MRI findings and histological features of resected bone specimens, bone oedema was scored again from the preoperative MRI scans of 2 of the original 9 patients and another 2 patients who had been recruited subsequently. Two different readers $(\mathrm{AD}$ and $\mathrm{BC})$, who were blinded to clinical and histopathological data, were introduced as the 2 original readers were unblinded by this stage. A modified system for scoring MRI bone oedema was introduced to capture intensity as well as the extent of its distribution in the localised region where bone was subsequently resected. The spatial extent of bone oedema was scored as follows: 0, 1, 2, 3 as per 0 (none), $1, \leqslant 33 \%, 2,34-66 \%$ and $3,67-100 \%$ of involved bone, based on RAMRIS. ${ }^{13}$ The intensity of bone oedema was scored as follows: Grade $0=$ none, Grade $1=$ mild to moderate, Grade $2=$ intense. The spatial and intensity scores were multiplied to give a final score (maximum possible $=6$ per site). In 3 patients, bone oedema could be assessed from T2W images. However, in 1 patient (patient No 10), MRI scan data were incomplete as fat saturation on $\mathrm{T} 2 \mathrm{w}$ images was unsuccessful. The presence/absence of bone oedema on the preoperative MRI scan from this patient was determined by the scoring radiologists from appearances on pre gadolinium Tlw images (bone oedema appears as low signal) and the corresponding postcontrast Tlw images where bone oedema appears as a high signal. ${ }^{14}$

\section{Histological studies}

A total of 7 bone specimens from 4 patients were available for histological analysis. Two samples were from 2 of the original 9 patients recruited (patients 6 and 7), and 2 were from new patients recruited to this part of the study (patients 10 and 11 ). For the latter 2 patients, bone oedema data were not analysed for surgical-site association, as recruitment occurred after the original analysis had been performed (and readers were unblinded). Bone samples were fixed in neutral buffered formal-saline, demineralised at room temperature in $10 \%$ formic acid for 1 week and embedded in paraffin. Three consecutive 3-micron sections were made 150 microns apart. Sections were mounted on glass slides and stained with haematoxylin and eosin. Specimens from patients 10 and 11 were sectioned in the coronal plane. The bone specimen from patient 6 (medial eminence of 1st metatarsal head) was sectioned parallel to the largest surface area. Information regarding the plane of section was not available for samples from patient 7. The pathologist (AK) reported the histology of the bone specimens blinded to MRI data.

\section{Statistical analysis}

Intraclass correlation coefficients (ICCs) were calculated for interobserver reliability of MRI scores. Scores from the 2 observers were averaged and analysed as ordinal variables, using a multinomial model. Generalised linear models were used to determine whether synovitis, bone erosion and bone oedema were more likely to be present at surgical than nonsurgical sites and whether increasing scores were associated with increasing likelihood of the site being surgical. Bone oedema and erosion data were dichotomised using cutoff points of $\geqslant 1.5$ for oedema (range 0 to 3 ) and $\geqslant 4$ for erosion (range 0 to 10). Regression models were used to investigate whether high-grade bone abnormalities were more likely within the surgical field. Pearson's correlations were calculated to assess the association between bone oedema (mean score across all sites for each patient) and scores for pain and CRP.

\section{RESULTS}

\section{Demographics}

Table 1 summarises demographics, medications, disease activity and orthopaedic procedures in the 11 patients recruited. Patients $1-9$ were included in Part 1 of the study investigating the association between bone oedema and surgical site. Bone was available from patients $6,7,10$ and 11 for histopathological correlation (Part 2). All patients were Caucasian, apart from one Indian female, and 9 were seropositive. Most had longestablished disease with a median disease duration of 24 years (range 6 to 43 years).

\section{Part 1. Investigating the association between MRI bone oedema and site of surgery}

MRI scans were initially available for 9 patients (table 1 , patients (-9), and these data were used to investigate whether bone oedema was more commonly found within the surgical zone than outside this region. Two observers scored scans for bone oedema, synovitis and bone erosion in a blinded fashion using the RAMRIS system. ${ }^{13}$

\section{Inter-observer reliability for MRI scoring}

ICCs for inter-observer reliability were 0.51 for bone oedema, 0.68 for erosion and 0.60 for synovitis, indicating moderate reliability despite advanced joint destruction and presence of artefact in several instances.

\section{Bone oedema, erosion and synovitis were more common within the surgical field}

For each patient, sites within the surgical field were assessed for erosion, bone oedema and synovitis. The median number of bone sites within the surgical field was 6 (range 2 to 11 ), while the number of sites outside was 20 (6 to 32). Bone oedema, erosions and synovitis were present at $60 \%, 75 \%$ and $70 \%$ of surgical and $38 \%, 41 \%$ and $31 \%$ of non-surgical sites, 
Table 1 Demographic, clinical and surgical details of all 11 patients

\begin{tabular}{|c|c|c|c|c|c|c|c|c|}
\hline \multicolumn{9}{|l|}{ Clinical scores } \\
\hline $\begin{array}{l}\text { Patient no./age } \\
\text { (years)/sex }\end{array}$ & Medications* & $\begin{array}{l}\text { Disease } \\
\text { duration } \\
\text { (years) }\end{array}$ & HAQ & DAS & ESR & CRP & $\mathrm{Sw} / \mathrm{Td}$ int & Type of surgical procedure \\
\hline $1 / 61 / \mathrm{F}$ & MTX, SSZ & 11 & 1.5 & 4.30 & 4 & N/A & $8 / 33$ & Right-wrist arthrodesis \\
\hline $2 / 61 / M$ & MTX, pred, LEF & 21 & 0 & N/A & N/A & N/A & $5 / 10$ & $\begin{array}{l}\text { Lapidus precedure left foot, modified Hibbs transfer, 3rd metatarsal shortening, 4th toe } \\
\text { PIP fusion, 5th toe flexor tenotomy }\end{array}$ \\
\hline $3 / 65 / F$ & MTX, pred, $\mathrm{HCQ}$ & 28 & 1.88 & 4.20 & 16 & 5 & $4 / 27$ & Arthrodesis 4 th and 5th tarsometatarsal joints \\
\hline $4 / 53 / \mathrm{F}$ & MTX, pred, LEF & 15 & 3.25 & 5.17 & 37 & 81 & $14 / 28$ & Arthroplasty right ring and middle PIP joints \\
\hline $5 / 59 / F$ & MTX & 25 & 4.25 & 2.96 & 11 & 7 & $5 / 9$ & $\begin{array}{l}\text { Right lapidus procedure, release and reduction MTP joints } 2 \text { and } 5 \text {, PIP joint fusion toes } \\
2-5\end{array}$ \\
\hline 6/64/F† & MTX, pred, HCQ & 43 & 0.63 & 2.87 & 4 & 2 & $5 / 12$ & Left 1st metatarsal osteotomy, right 4th toe PIP arthrodesis \\
\hline 7/57/F† & MTX, pred, SSZ HCQ & 15 & 2.88 & 5.87 & 12 & 14 & $26 / 34$ & Bilateral forefoot arthroplasties (resection of metatarsal heads $2-5$, fusion 1st MTP joint) \\
\hline $8 / 77 / \mathrm{F}$ & Pred, cyclo & 6 & 2.38 & 5.75 & 53 & 32 & $21 / 28$ & Arthrodesis left naviculocuneiform, intercuneiform, 2nd and 3rd TMT joints \\
\hline $9 / 59 / \mathrm{F}$ & MTX, pred, SSZ & 29 & 2.75 & 3.12 & 43 & 34 & $2 / 8$ & Right foot 1st MTP fusion and excision of lesser metatarsal heads 2-5 \\
\hline 10/49/Ft & SSZ, pred, HCQ, MTX & 26 & 1.88 & 5.29 & 8 & 4 & $32 / 22$ & Arthroplasty left hand, MCP joints $2-5$ \\
\hline $11 / 50 / \mathrm{Ft}$ & $\mathrm{HCQ}$ & 24 & 1.88 & 3.57 & 31 & 30 & $14 / 8$ & Left metatarsal scarf osteotomy, osteotomies 2nd and 4th MTP joints \\
\hline
\end{tabular}

*Medications: all patients on NSAIDs, MTX, methotrexate; pred, prednisone (10 mg or less); HCQ, hydroxychloroquine; SSZ, sulfasalazine; LEF, leflunomide; cyclo, ciclosporine; thistopathology available; see table 2. DAS, disease activity score; ESR, erythrocyte sedimentation rate $(\mathrm{mm} / \mathrm{h})$; HAQ, health assessment questionnaire; PIP, proximal interphalangeal joints; MTP, metatarsophalangeal joints; Sw/Td, swollen and tender joints; N/A, not available.

respectively. Higher degrees of bone oedema were found with increasing likelihood at surgical compared with non-surgical bone sites $(\mathrm{OR}=3.12(1.96$ to 4.97$) \mathrm{p}<0.0001)$ and the same applied to bone erosion to a lesser extent $(\mathrm{OR}=1.28$ ( 1.11 to 1.47) $\mathrm{p}=0.0006)$. High scores for synovitis were also more common at joints within the surgical field $(\mathrm{OR}=2.45$ ( 1.5 to $4.5), \mathrm{p}=0.0005)$.

\section{High-grade MRI bone oedema was found frequently within the surgical field}

In several patients, there was extensive bone oedema exactly at the site of intended surgery on the preoperative MRI scan. Examples are shown for patients 8, 6 and 4 in fig 1 (A-I). For patient 4, a sagittal T2W MRI image is shown to illustrate bone oedema involving the base of the middle phalanx (panel I), as a coronal $\mathrm{T} 2 \mathrm{~W}$ view was not available. However, a coronal Tlw postcontrast VIBE image also confirmed bone oedema at this site (available on request). Regression analyses using cutoff points of $\geqslant 1.5$ for bone oedema (score $50 \%$ of maximum or greater) and $\geqslant 4$ for erosion (score $40 \%$ of maximum or greater) revealed that high-grade bone oedema was very likely to be present at a surgical site, with an odds ratio (OR) of 9.3 (3.5 to 24.2), $\mathrm{p}<0.0001$. High degrees of bone erosion were associated with surgical site to a lesser degree; $\mathrm{OR}=4.1$ (1.4 to 12.0$)$, $\mathrm{p}<0.0097$. Bone oedema scores were correlated with pain scores in these patients $(\mathrm{r}=0.67, \mathrm{p}=0.048)$ and strongly correlated with CRP $(\mathrm{r}=0.86, \mathrm{p}=0.01)$.

\section{Part 2. Comparing MRI bone oedema with histology}

Seven bone samples from 4 patients were available for histopathological analysis and comparison with MRI bone oedema from preoperative MRI scans (table 2).

Scoring MRI bone oedema (resected bony sites only) Two new readers ( $\mathrm{AD}$ and $\mathrm{BC}$ ) who were blinded to clinical details were introduced for the direct comparison between MRI bone oedema and histology, as the original 2 readers were now unblinded. It was also decided to modify the MRI bone oedema

Table 2 Comparison between preoperative MRI bone oedema score* and description of histopathology for resected bone specimens

\begin{tabular}{|c|c|c|c|c|}
\hline \multirow[b]{2}{*}{ Patient no. } & \multirow[b]{2}{*}{ Bone specimen } & \multicolumn{2}{|c|}{ MRI bone oedema score } & \multirow[b]{2}{*}{ Histopathology report } \\
\hline & & Reader 1 & Reader 2 & \\
\hline 6 & $\begin{array}{l}\text { Left } 1 \text { st metatarsal head, } \\
\text { medial eminence }\end{array}$ & 6 & 6 & $\begin{array}{l}\text { Moderately intense patchy lymphoplasmacytic infiltration in the subchondral marrow, patchy } \\
\text { pannus formation over the articular cartilage and subchondral cyst }\end{array}$ \\
\hline 7 & Right 5th metatarsal head & 2 & 2 & $\begin{array}{l}\text { Thinning and focal necrosis of articular cartilage with marginal pannus formation with patchy } \\
\text { aggregations of mature small lymphocytes in the subchondral region, plus subchondral cyst }\end{array}$ \\
\hline \multirow[t]{4}{*}{10} & Left 2nd metacarpal head & 0 & 0 & $\begin{array}{l}\text { Degeneration and focal loss of cartilage and thin fibrovascular pannus formation, focal } \\
\text { remodelling of subchondral bone with light mononuclear cell infiltration and slight marrow } \\
\text { fibrosis }\end{array}$ \\
\hline & Left 3rd metacarpal head & 0 & 0 & $\begin{array}{l}\text { Patchy loss of articular cartilage with thin fibrous pannus formation, irregular interface } \\
\text { between articular cartilage and subchondral bone with prominent congested capillaries but } \\
\text { no evidence of osteitis }\end{array}$ \\
\hline & Left 4 th metacarpal head & 0 & 0 & $\begin{array}{l}\text { Degeneration of articular cartilage with thin fibrous pannus formation at joint margin, slight } \\
\text { osteitis }\end{array}$ \\
\hline & Left 5th metacarpal head & 0 & 0 & $\begin{array}{l}\text { Degeneration of articular cartilage, thin fibrous pannus formation with slight focal osteitis and } \\
\text { some remodelling of subchondral bone }\end{array}$ \\
\hline 11 & Left 1st metatarsal head & 1 & 2 & $\begin{array}{l}\text { Focal disruption of the articular cartilage and a flask-shaped defect extending into the } \\
\text { medullary space; the lining is composed of fibrous granulation tissue with telangiectatic new } \\
\text { blood vessels and slight lymphocyte infiltration; elsewhere the normal articular cartilage is } \\
\text { replaced by fibrous pannus and fibrocartilage }\end{array}$ \\
\hline
\end{tabular}

*MRI bone oedema scored separately by 2 radiologists according to the extent and intensity of bone oedema at the site where bone was resected as follows: spatial extent of bone oedema; Grade 0 for none, Grade 1 for $\leqslant 33 \%$, Grade 2 for $34-66 \%$, Grade 3 for $67-100 \%$ of area of bony site assessed. Intensity of bone oedema; Grade $0=$ none, Grade $1=$ moderate intensity, Grade $2=$ high intensity. Total score comprises spatial extent $\times$ intensity scores. Maximum possible score per site $=6$. 

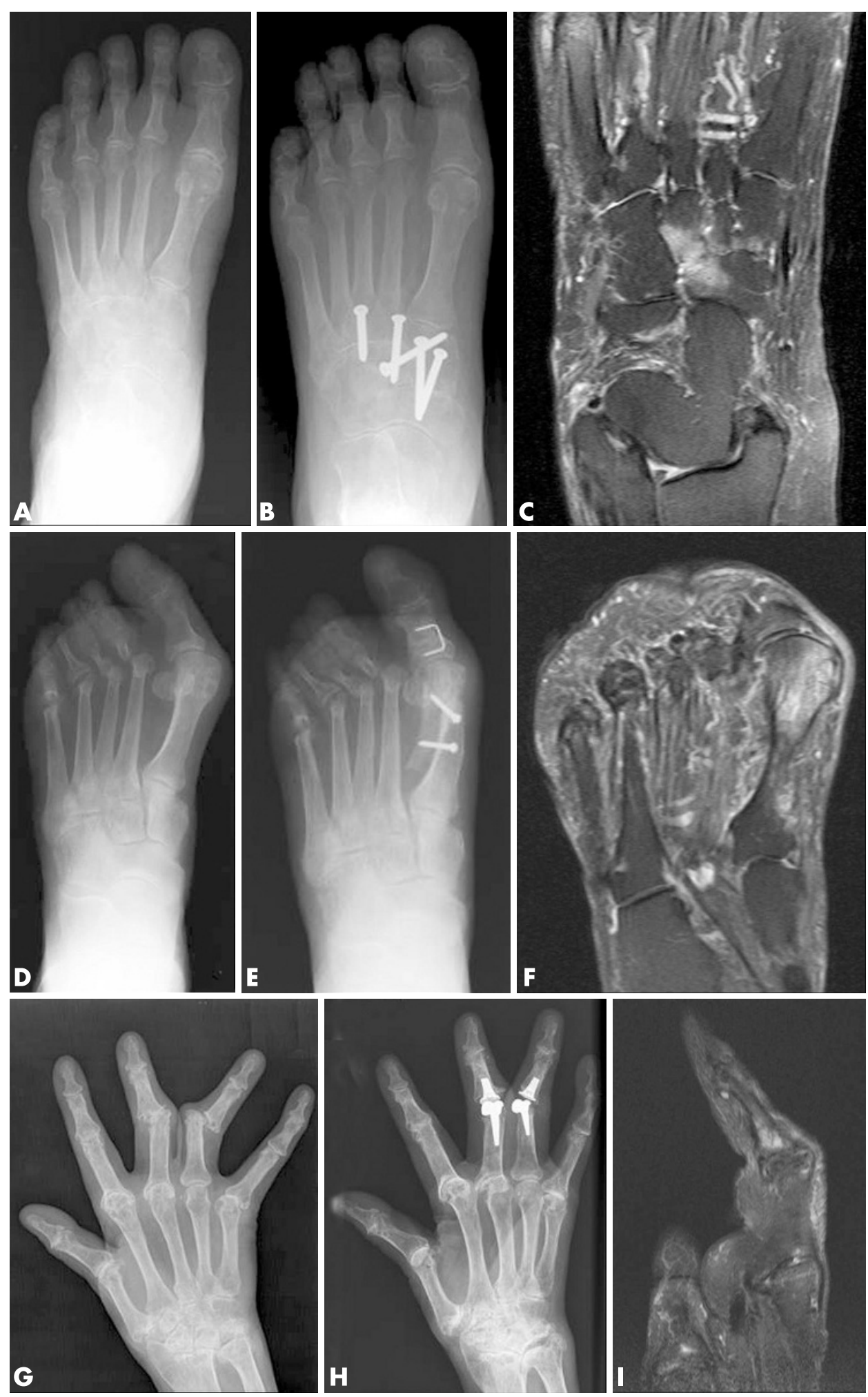

Figure 1 Bone oedema was seen frequently on the preoperative MRI scan within the field of intended surgery. (A) Patient 8.

Preoperative radiograph of left foot showing reduced joint space at the tarsometatarsal joints and likely erosions "en face". (B) Postoperative radiograph showing screws across naviculocuneiform and

tarsometatarsal joints for arthrodesis. (C) Preoperative MRI scan of the foot (T2w coronal image) showing extensive bone oedema involving the medial, middle and lateral cuneiforms and navicular. (D) Patient 6. Preoperative radiograph of left foot showing severe hallux valgus and resected metatarsal heads (previous surgery). (E) Postoperative radiograph showing that a scarf osteotomy has been performed at the 1 st metatarsal to improve alignment. (F) Preoperative MRI scan (T2w coronal image) showing extensive bone oedema involving the head of the 1 st metatarsal extending to the mid-shaft. (G) Patient 4. Preoperative radiograph of right hand showing advanced erosive damage of wrist, MCP and PIP joints with dislocation of $3 \mathrm{rd}$ and 4 th PIP joints. (H) Postoperative radiograph showing joint replacements at $3 \mathrm{rd}$ and 4 th PIP joints. (I) MRI scan of the 3rd finger of the right hand (sagittal T2w image) showing intense bone oedema involving the base of the middle phalanx and extending to the mid-shaft. scoring system as only small regions were being scored (where bone had been resected), and a score for intensity of bone oedema was added as this was felt most likely to equate with cellular density. The total bone oedema score was the product of scores for its spatial extent and intensity as described in the Methods section. The scores for both readers were identical for every patient except Patient 11 where there was a difference of one point (table 2). The interobserver reliability ICC for these scores was 0.985 .
Comparing MRI bone oedema at resected sites with histopathology

Results are shown in table 2. As the number of histological samples was small, it was decided not to attempt to score these changes, but the pathologist's report $(\mathrm{AK})$ is presented for comparison with the MRI bone oedema scores. In 4 of the 7 samples, there was complete concordance for the presence and severity of MRI bone oedema and the description of osteitis in bone samples. Figures $2 \mathrm{a}-\mathrm{c}$ show the histopathology and 
corresponding MRI appearance for 3 of these specimens (patients 6, 7 and 10). In patient 6 (fig 2a), there was extensive replacement of trabecular subchondral bone by cellular and myxoid tissue. The MRI scan of the resected region (medial eminence of the lst metatarsal head) was graded as 6 for bone oedema (maximum) by both readers. For Patient 10, 2nd metatarsal head (fig 2b), there was no bone oedema on the pathological section, and none was scored from MRI scans. For Patient 7 (fig 2c), there were "patchy aggregations" of lymphocytes in the subchondral region, and both radiologists scored bone oedema as present but low positive. The only instances where there was a lack of concordance between a report of osteitis and a positive score for MRI bone oedema was in Patient 10, where all MRI scans were scored as 0 , but 3 samples were reported as showing "slight osteitis".

\section{DISCUSSION}

To our knowledge, this is the first report to investigate the distribution of MRI bone oedema in RA patients selected for reparative orthopaedic surgery. The study was designed to investigate, first, whether bone oedema was present on preoperative MRI scans, in the regions selected for surgery. Second, where bone samples were resected at the time of the operation, we aimed to determine whether preoperative MRI bone oedema was associated with the presence of a cellular infiltrate or osteitis in subchondral bone. Our findings confirmed first that there was an association between the presence of bone oedema and the site of intended surgery. High-grade bone oedema was 9 times more likely to be scored within the surgical field than outside it and was more strongly associated with the site of surgery than MRI bone erosion. As this site was predefined by the orthopaedic surgeon without knowledge of MRI scan results, this suggests an association between factors influencing the site of surgery and MRI bone oedema.

The decision to undertake orthopaedic surgery in RA patients can be influenced by cosmetic and functional considerations, but the overwhelming driver is to relieve patients' pain. ${ }^{15}$ Traditionally, it has been assumed that pain derives from synovitis, bone erosion or secondary osteoarthritis. Our findings challenge this assumption. While we found synovitis and erosion within the surgical field as expected, the new and surprising observation was that bone oedema was so common and relatively severe. Moreover, the bone oedema score was associated with the pain score as well as the CRP. Bone oedema could be secondary to synovitis and merely indicate "bone reaction" to overlying synovial inflammation, ${ }^{16}$ but if so, it is surprising that its association with surgical site was so strong and more apparent than with synovitis. A second explanation posits that bone oedema reflects a process of osteitis within subchondral bone that is pre-erosive and occurs separately to, but often in conjunction with, synovitis. ${ }^{10}$ This accords with our own observations that bone oedema was the strongest individual predictor of bone damage and functional impairment in an RA cohort where it was detected in $40-60 \%$ of patients over an 8 -year period and was consistently associated with pain. ${ }^{3}{ }^{17}$

What is the clinical significance of the apparent association between MRI bone oedema and the site of surgery in these RA patients? We feel this lies in the implication that an active, painful and potentially pre-erosive process of osteitis could be occurring in the regions targeted for surgery. If our observation can be verified in other studies, it is not inconceivable that surgeons could in the future use the presence and distribution of bone oedema to decide on the best site for joint surgery aimed at pain relief for the patient. In future studies, it would be very interesting to obtain postoperative MRI scans to see whether bone oedema had regressed where pain was relieved after surgery, and we intend to arrange follow-up scans for our patients to investigate this. We would also suggest that rheumatologists consider the presence of MRI bone oedema as an indicator of active bone disease and a reason to intensify medical management with disease-modifying antirheumatic drugs (DMARDs) or biological therapies, as recent studies have confirmed that bone oedema can be reduced by anti-TNF agents, coinciding with clinical improvement. ${ }^{18} 19$

To further explore the possibility that MRI bone oedema represents a cellular infiltrate within the subchondral marrow, we compared scores on preoperative MRI scans with histopathological appearances of bone resected at the time of surgery. Recruitment proved difficult, and over 18 months we were only able to enrol 11 patients. In many of these, anatomically preserved bone samples were not available for analysis due to the surgical technique used (often resulting in shaving or "morcellising" bone). This reflects both an overall reduction in surgical interventions in RA patients as described by Weiss et $a^{20}$ and a move towards more limited procedures involving less bone resection, particularly in forefoot surgery. ${ }^{21}$ In 2 patients from the original 9, suitable samples were available, and 2 further patients were enrolled to extend this aspect of the study. Our results confirmed those of others indicating that MRI bone oedema does correspond with regions where there is a cellular infiltrate replacing marrow fat in subchondral trabecular bone. As described by Jimenez-Boj et al, ${ }^{6}$ we found that infiltrates could take 2 forms; first, the extension of synovial tissue through the subchondral plate, which had been breached by an erosion, and second, separate foci of lymphoid cells, which were not associated with erosions. In 4 of the 7 bone samples examined, there was complete concordance between the presence and severity of MRI bone oedema and the degree of osteitis. In 3 samples, where there was no MRI bone oedema observed, only "slight focal" osteitis was detected. These findings suggest a "threshold" phenomenon for MRI bone oedema as described by Appel et al ${ }^{22}$ due to the much greater image resolution of histopathology compared with MRI scanning.

It could be argued that our findings simply reflect the presence of secondary $\mathrm{OA}$ in these patients with advanced rheumatoid disease. While OA is likely to be present, the histopathological features of each specimen were typical of RA, ranging from active inflammation to a burnt-out appearance with fibrous pannus. Certainly, MRI bone oedema has been described in OA joints, ${ }^{23}$ but in general it tends to be less florid than in the examples shown here. Unfortunately, teasing out the distinction between the bone oedema of RA and that associated with secondary $\mathrm{OA}$ is unlikely to be possible in humans as bone specimens are usually only available from patients with advanced disease (disease duration in the majority of our patients was $>10$ years). However, the work of Proulx et $a l^{9}$ has indicated that MRI bone oedema corresponds with inflammatory infiltrates in subchondral bone in a TNF-transgenic mouse model of arthritis, and it may be possible to extend those animal studies to investigate early disease.

If osteitis in subchondral bone is part of the pathology of RA, it becomes important to characterise the cells within these foci of inflammation. Bugatti et al described B and T lymphocytes in subchondral lymphoid aggregates and described a correlation between the intensity of this infiltrate and the number of associated osteoclasts. ${ }^{8}$ Watson et al described CD45RO T cells and CD20 B cells in germinal centres within subchondral bone in a similar group of RA patients ${ }^{24}$ and suggested that these cells were likely to be immunologically active, possibly responding to local antigen. Bollow et $a^{25}$ also described cellular 

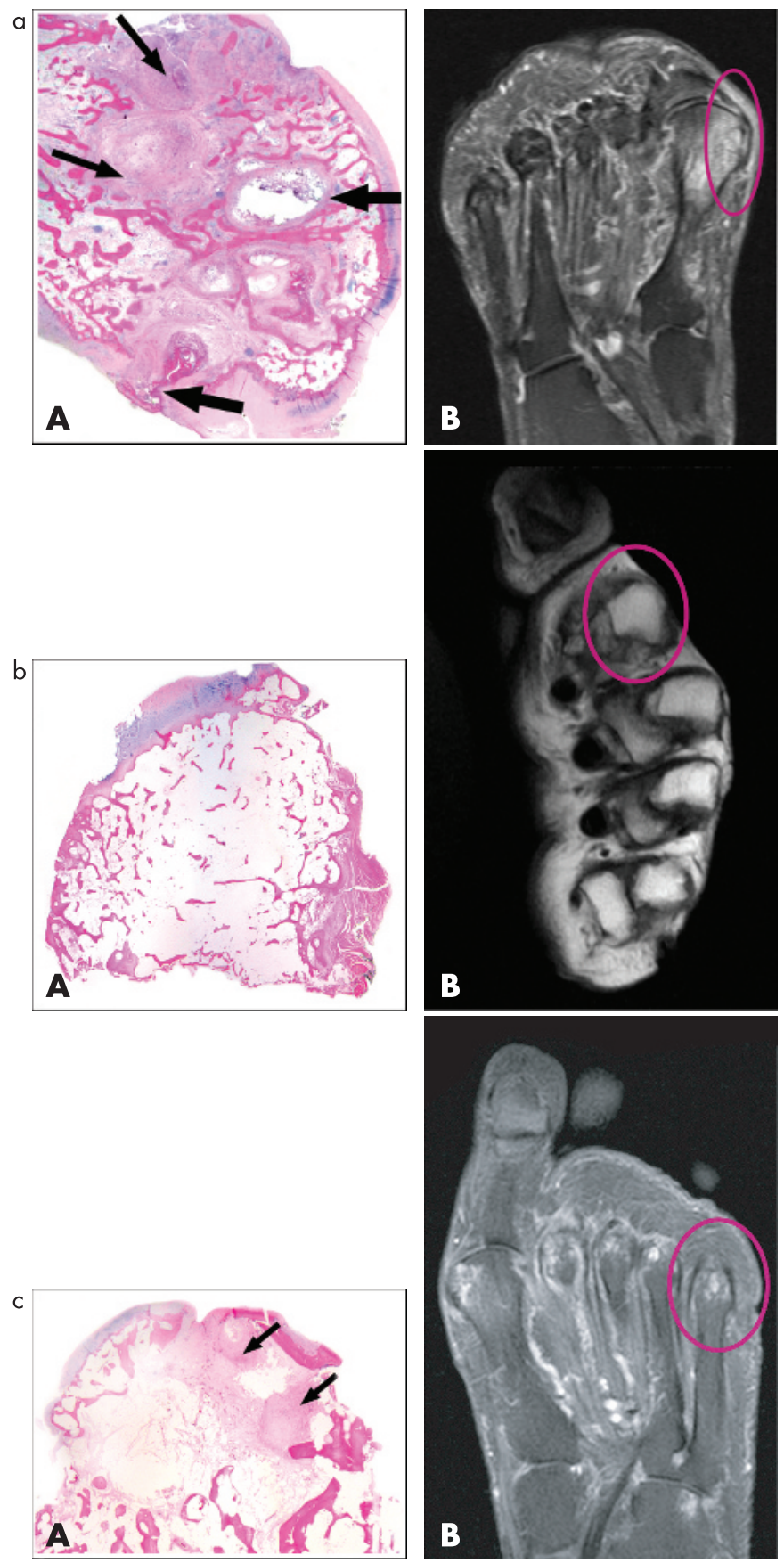

Figure 2 (a) Patient 6. (A) Histopathological specimen: resected medial eminence, left great toe, sagittal section, shows infiltration of bone (replacement of fat) by cellular and myxoid tissue (thin arrows) plus erosions (wide arrows). (B) Corresponding MRI scan (coronal T2w fat sat image) showing extensive bone oedema involving the entire metatarsal head including the medial eminence. (b) Patient 10. (A) Histopathological specimen: head of 2 nd left metatarsal showing no osteitis. (B)

Corresponding MRI scan (axial T1w image without contrast) not showing bone oedema. (c) Patient 7. (A) Histopathological specimen: head of 5th metatarsal right foot showing extensive replacement of marrow fat in subchondral region by fibrous tissue and cellular infiltrates (arrows). (B) MRI scan (coronal T2w fat sat image) showing bone oedema and erosion involving the right 5 th metatarsal head.

infiltrates containing activated lymphocytes and fibroblasts in bone biopsy specimens from sacroiliac joints of patients with spondyloarthropathies. They found that cell counts correlated with the extent of MRI bone oedema. We are currently pursuing immunohistochemical studies of the bone resected from our patients to characterise the phenotype and activation status of cells present within foci of marrow inflammation as well as their association with subchondral osteoclasts.

There are a number of shortcomings to this study. Due to small patient numbers, we were unable to stratify data to investigate the possible influence of DMARDs. However, it is significant that none of our patients were on anti-TNF agents, as these have been shown to reduce bone oedema in inflammatory arthritis. ${ }^{18} 19$ Ideally, 2-plane MRI information should have been available for all regions scanned. We acknowledge that this may have introduced bias but feel that the association between MRI bone oedema and the site of surgery was so strong that this is still likely to be a real finding. Inter-reader reliability for bone oedema scores in Part 1 of the study was only moderate, and was lower than in some studies using the RAMRIS score ${ }^{26}$ but not others. ${ }^{27}$ This was not surprising, as some joint regions had been largely destroyed by the rheumatoid process, and artefact was present in several instances. Part 2 of the study was complicated by difficulties obtaining specimens, and in some instances $\mathrm{T} 2 \mathrm{w}$ data were not available as fat saturation failed. Bone oedema was therefore scored by both radiologists from Tlw pre- and postcontrast images, but in these situations, scores were concordant, lending credence to these results.

In summary, we feel we have presented compelling evidence that MRI bone oedema in RA patients is associated with an inflammatory cellular infiltrate (osteitis) involving subchondral bone. We suggest that this osteitic lesion is painful and possibly associated with ongoing bony destruction, and that is the reason for its over-representation within the surgical field for patients undergoing reparative orthopaedic surgery. Further examination of the cells contributing to this subchondral infiltrate is indicated to better define their role in contributing to rheumatoid bony damage.

\section{ACKNOWLEDGEMENTS}

We wish to acknowledge the contribution of the orthopaedic surgeons who referred patients for this study: $\mathrm{Mr} \mathrm{M}$ Tomlinson, Mr Clayton Brown, Mr S Mills, Mrs H Rawlinson, Mr C Taylor, Mr A Hardy, Mr R Gordon and Mr J Cullen. We are grateful to Shelley Park and Sandra Winsor of the Centre for Advanced MRI and Sue Yeoman, Pam Antill, Cecilia Tong and Karen Pui of the Department of Rheumatology, Auckland City Hospital for their contributions. Special thanks to $\mathrm{Mr}$ Jonathan Ellison, Medical Photography, Greenlane Clinical Centre for assistance in preparation of the images. Supported by grants from Arthritis New Zealand, The Auckland Hospital Rheumatology Research Fund and the Megan Wynn Trust (funded studentship for GS).

\section{Authors' affiliations}

F M McQueen, A Gao, M Østergaard, A King, G Shalley, E Robinson, A Doyle, B Clark, N Dalbeth, Department of Molecular Medicine and Pathology, Faculty of Medicine and Health Sciences, University of Auckland, Park Rd, Auckland, New Zealand

Competing interests: None.

\section{REFERENCES}

1 Mottonen TT. Prediction of erosiveness and rate of development of new erosions in early rheumatoid arthritis. Ann Rheum Dis 1988;47:648-53.

2 Ostendorf B, Scherer A, Modder U, Schneider M. Diagnostic value of magnetic resonance imaging of the forefeet in early rheumatoid arthritis when findings on imaging of the metacarpophalangeal joints of the hands remain normal. Arthritis Rheum 2004;50:2094-102.

3 McQueen FM, Benton N, Perry D, Crabbe J, Robinson E, Yeoman S, et al. Bone edema scored on magnetic resonance imaging scans of the dominant carpus at presentation predicts radiographic joint damage of the hands and feet six years later in patients with rheumatoid arthritis. Arthritis Rheum 2003:48:1814-27.

4 Benton N, Stewart N, Crabbe J, Robinson E, Yeoman S, McQueen FM. MRI of the wrist in early rheumatoid arthritis can be used to predict functional outcome at 6 years. Ann Rheum Dis 2004;63:555-61. 
5 Tamai M, Kawakami A, Takao S, Uetani M, Arima K, Tanaka F, et al. Bone marrow oedema determined by MRI reflects severe disease status in patients with early-stage rheumatoid arthritis. Ann Rheum Dis, 2006;65(Suppl II), 629.

6 Jimenez-Boj E, Nöbauer-Huhmann I, Hanslik-Schnabel F, Dorotka R, Wanivenhaus $A$, Kainberger $F$, et al. Bone erosions and bone marrow edema as defined by magnetic resonance imaging reflect true bone marrow inflammation in rheumatoid arthritis. Arthritis Rheum 2007;56:1118-24.

7 Barrie HJ. Histologic changes in rheumatoid disease of the metacarpal and metatarsal heads as seen in surgical material. J Rheumatol 1981:8:246-57.

8 Bugatti S, Caporali R, Manzo A, Vitolo B, Pitzalis C, Montecucco C. Involvement of subchondral bone marrow in rheumatoid arthritis: lymphoid neogenesis and in situ relationship to subchondral bone marrow osteoclast recruitment. Arthritis Rheum 2005:52:3448-59.

9 Proulx S, Kwok E, Shealy D, Ritchlin C, Schwarz E. Understanding bone marrow edema in arthritis: 3D-MRI \& histology analyses of TNF-Tg Mice. Arthritis Rheum 2006;54(Suppl):S798-9.

10 McQueen FM, Ostendorf, B. What is MRI bone oedema in rheumatoid arthritis and why does it matter? Arthritis Res Ther 2006;8:222-5.

11 Arnett FC, Edworthy SM, Bloch DA, McShane DJ, Fries JF, Cooper NS, et al. The American Rheumatism Association 1987 revised criteria for the classification of rheumatoid arthritis. Arthritis Rheum 1988;31:315-24.

12 van der Heijde DM, van't Hof MA, van Riel PL, Theunisse LA, Lubberts EW, van Leeuwen $M A$, et al. Judging disease activity in clinical practice in rheumatoid arthritis: first step in the development of a disease activity score. Ann Rheum Dis 1990;49:916-20.

13 Østergaard M, Peterfy C, Conaghan P, McQueen F, Bird P, Ejbjerg B, et al. OMERACT rheumatoid arthritis magnetic resonance imaging studies. Core set of MRI acquisitions, joint pathology definitions, and the OMERACT RA-MRI scoring system. J Rheumatol 2003;30:1385-6.

14 Stewart NR, Crabbe JP, McQueen FM. Magnetic resonance imaging of the wrist in rheumatoid arthritis: demonstration of progression between 1 and 6 years. Skel Radiol 2004:33:704-11.

15 Dunbar RP, Alexiades MM. Decision making in rheumatoid arthritis. Determining surgical priorities. Rheum Dis Clin North Am 1998;24:35-54.

16 McGonagle D, Conaghan PG, O'Connor P, Gibbon W, Green M, Wakefield R, et al. The relationship between synovitis and bone changes in early untreated rheumatoid arthritis: a controlled magnetic resonance imaging study.[see comment]. Arthritis Rheum 1999;42:1706-11.

17 McQueen FM, Stewart N, Crabbe J, Robinson E, Yeoman S, Tan PL, et al. Magnetic resonance imaging of the wrist in early rheumatoid arthritis reveals a high prevalence of erosions at four months after symptom onset. Ann Rheum Dis 1998:57:350-6.

18 Haavardsholm E, Østergaard M, Schildvold A, Kvien T. MRI findings reflecting inflammation is more responsive than clinical measures of disease activity when monitoring anti-TNF alpha treatment in RA patients. Arthritis Rheum 2006;54(Suppl):S800.

19 Marzo-Ortega H, McGonagle D, Rhodes LA, Tan AL CP, O-Connor P, Tanner SF, et al. Efficacy of infliximab on MRI determined bone oedema in psoriatic arthritis. Ann Rheum Dis. Published Online First: 21 December 2006. doi:10.1136/ ard.2006.063818.

20 Weiss RJ, Stark A, Wick MC, Ehlin A, Palmblad K, Wretenberg P. Orthopaedic surgery of the lower limbs in 49,802 rheumatoid arthritis patients: results from the Swedish National Inpatient Registry during 1987 to 2001. Ann Rheum Dis 2006;65:335-41.

21 Toolan BC, Hansen ST Jr. Surgery of the rheumatoid foot and ankle. Curr Opin Rheumatol 1998;10:116-9.

22 Appel H, Loddenkemper C, Grozdanovic Z, Ebhardt H, Dreimann M, Hempfing $A$, et al. Correlation of histopathological findings and magnetic resonance imaging in the spine of patients with ankylosing spondylitis. Arthritis Res Ther 2006;8:R143.

23 Felson DT, Chaisson CE, Hill CL, Totterman SM, Gale ME, Skinner KM, et al. The association of bone marrow lesions with pain in knee osteoarthritis. [see comment]. Ann Intern Med 2001;134:541-9.

24 Watson WC, Tooms RE, Carnesale PG, Dutkowsky JP. A case of germinal center formation by CD45RO T and CD20 B lymphocytes in rheumatoid arthritic subchondral bone: proposal for a two-compartment model of immune-mediated disease with implications for immunotherapeutic strategies. Clin Immunol Immunopathol 1994;73:27-37.

25 Bollow M, Fischer T, Reisshaver H, Backhaus M, Sieper J, Hamm B, et al. Quantitative analyses of sacroiliac biopsies in spondyloarthropathies: T cells and macrophages predominate in early and active sacroiliitis-cellularity correlates with the degree of enhancement detected by magnetic resonance imaging. Ann Rheum Dis 2000;59:135-40.

26 Haavardsholm EA, Ostergaard M, Ejbjerg BJ, Kvan NP, Uhlig TA, Lilleas FG, et al. Reliability and sensitivity to change of the OMERACT rheumatoid arthritis magnetic resonance imaging score in a multireader, longitudinal setting. Arthritis Rheum 2005;52:3860-7.

27 Bird P, Ejbjerg B, Lassere M, Østergaard M, McQueen F, Peterfy C, et al. A multireader reliability study comparing conventional, high field MRI with extremity, low field MRI in rheumatoid arthritis. J Rheum. In press.

\section{Submit an eLetter, and join the debate}

eLetters are a fast and convenient way to register your opinion on topical and contentious medical issues. You can find the "submit a response" link alongside the abstract, full text and PDF versions of all our articles. We aim to publish swiftly, and your comments will be emailed directly to the author of the original article to allow them to respond. eletters are a great way of participating in important clinical debates, so make sure your voice is heard. 